\title{
Pseudosymmetry in EBSD Patterns
}

Mark D. Vaudin, MSEL, National Institute of Standards and Technology, Gaithersburg, MD 20899

Automatic indexing of electron backscatter diffraction (EBSD) patterns depends on the successful application of algorithms that: (1) determine the position (and to a lesser degree the intensity) of Kikuchi bands in EBSD patterns (typically using a Hough or Radon transform); (2) for each band detected, determine the plane that contains the center line of the band and the point where the electron beam strikes the sample; (3) calculate the angles between these experimentally determined planes taken over all possible pairs; and (4) determine which of the phases preselected by the user can best match the experimental angles determined in (3), and thus assigns an orientation to the diffracting crystal and an indexing scheme to the recorded pattern. The ever-increasing speed of the computers and detectors has lead to the current success of this approach, with very high speeds being obtained, of the order of 100 orientations per second being measured under the best conditions (see [1] for a full discussion of EBSD and its applications).

There are occasions when two indexing solutions appear to be equally likely according to the algorithms employed to perform the indexing. One of the reasons this occurs is pseudosymmetry, where two indexing schemes cannot be distinguished because an n-fold rotation axis in a crystal structure is detected as a $2 \mathrm{n}$-fold axis, so that there are two variants that are equally likely. This is either due to the failure to detect the diffraction features that would distinguish between the variants, or the failure to detect line positions with sufficient accuracy to distinguish small differences in the ratios of unit cell dimensions. An example of the first problem is a 3-fold axis detected as 6-fold, for instance $<0001>$ in trigonal $\mathrm{Al}_{2} \mathrm{O}_{3}$; an example of the second is a 2-fold axis detected as 4-fold, for instance [100] or [010] seen as [001] in a tetragonal material where $\mathrm{c} / \mathrm{a} \approx 1$.

Figure 1 illustrates two different problems in indexing $<0001>$ EBSD patterns from alumina, including distinguishing a 6-fold axis from a 3-fold axis. Three EBSD patterns from positions $1 \mu \mathrm{m}$ apart in the same grain are shown in Fig. 1(a) to (c). During an automated high speed scan the indexing schemes shown in Fig. 1(d) to (f) were ascribed, indicating the crystal orientations shown schematically in Fig. 1(g) to (i) with the crystal a-axes marked with red sticks and the respective Euler angles $\left(\varphi_{1}, \varphi, \varphi_{2}\right)$ listed below. Patterns (a) and (b) demonstrate the 6-fold/3-fold problem: the crystal schematic indicates the two indexing schemes are related by a rotation of $60^{\circ}$ about [0001], as does the $60^{\circ}$ change in $\varphi_{2}$. The regions of the two patterns containing the distinguishing features are circled in 1(d) and (e). There is a zone axis that is common to both circles, of the $<1-101>$ type, and the diffraction lines forming this zone axis are well observed, but this zone axis occurs with 6fold symmetry around the $<0001>$ axis. In the correct indexing scheme in (fig. 1(e)) there is a second zone axis in the circle which has 3-fold symmetry but the lines that intersect obliquely at the axis are weak and it is the failure to reliably detect these lines that leads to the erratic indexing. Figure 1(c) is an example of a pattern which has been indexed to give an orientation rotated $30^{\circ}$ about [0001] to the correct one. This typically happens when only 6 lines are detected by the Hough transform, passing through the [0001] axis with a 6-fold arrangement of these lines. It has been found that for specific patterns, increasing the number of lines used by the indexing algorithm can improve the likelihood of correctly indexing the pattern, but overall it typically leads to an increased number of failed indexings and the hit rate goes down. Grains of different orientations will result in different 
patterns on the detector, and in the area mapped this grain was the only one to give a substantial number of misindexings (40 points: 8 misindexings by $60^{\circ} ; 15$ misindexings by $30^{\circ}$.

The alumina orientation which produces an $<0001>$ zone axis near the center of the pattern is the most challenging to analyze unambiguously, because there is strong 6-fold symmetry to the pattern with only weak lines to break that symmetry and indicate that the axis is 3-fold. Another pseudosymmetry situation occurs when unit cell dimensions in tetragonal and orthorhombic symmetry materials are similar so that it is difficult to distinguish between, for example, the [100] and [001] directions in a tetragonal perovskite such as $\mathrm{PbZr}_{\mathrm{x}} \mathrm{Ti}_{1-\mathrm{x}} \mathrm{O}_{3}(\mathrm{PZT})$, where c/a-1 $=0.03$ at most. Distinguishing the axes does not depend on the presence or absence of relatively weak lines in the pattern, but on small differences in line position. The location of the c-axis in ferroelectric materials such as PZT is a technologically important measurement, and the effects of various experimental parameters on the ability to do this is being studied. Removing pattern distortions, improving the accuracy of detector calibration and assessing the effects of pattern noise are some of the avenues of research being pursued .

\section{References}

[1] V. Randle and O. Engler, Introduction to Texture Analysis, Gordon and Breach Science Publishers, Amsterdam, 2000

\section{Figure 1}
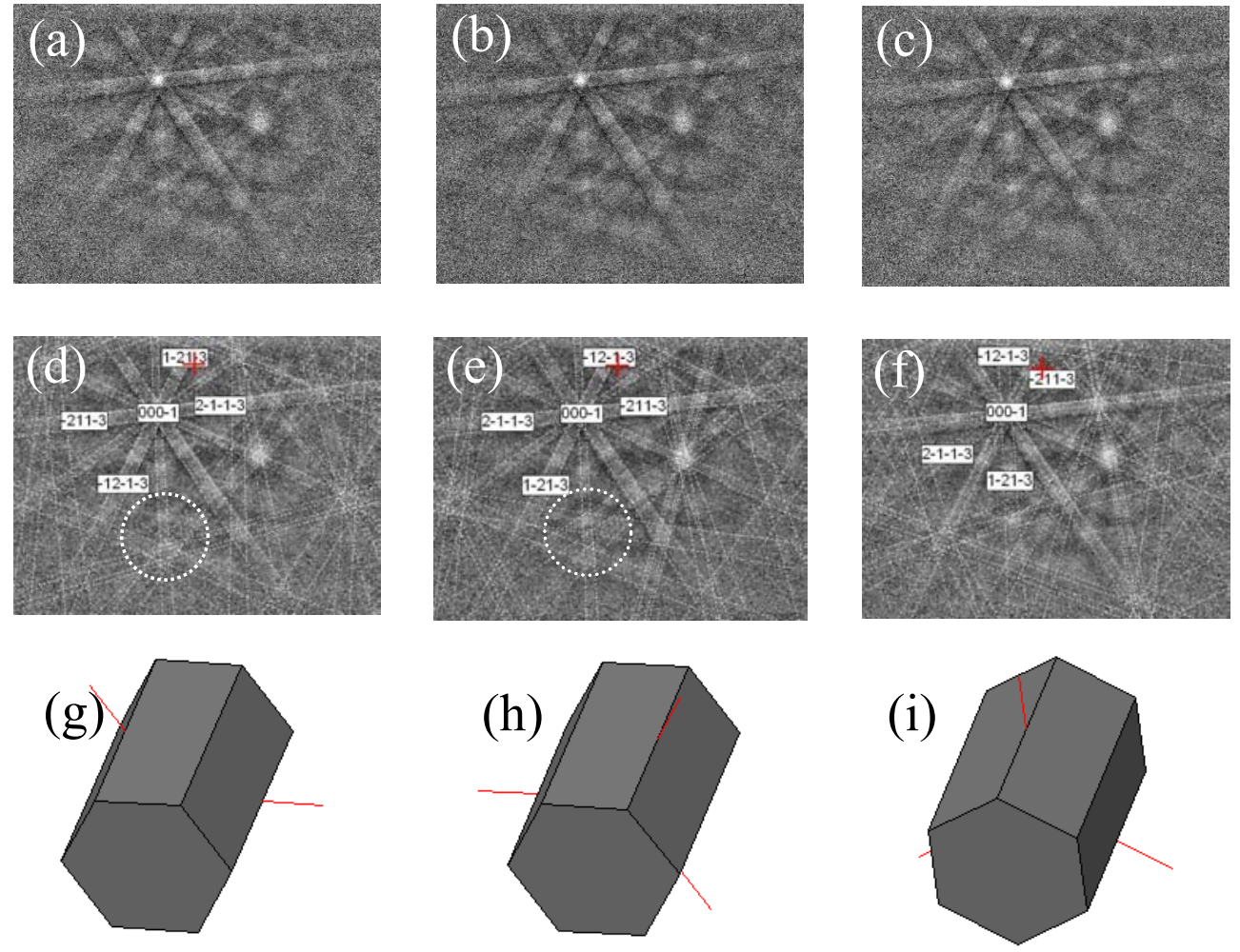

$156.4,141.0,64.8 \quad 156.8,140.7,4.7 \quad 157.4,141.2,95.2$ 